\title{
Elementos psicopedagógicos en niños de preescolar y factores de protección
}

\section{Psycho-pedagogical elements in preschool children and protection factors}

\author{
PACHECO-AMIGO, Beatriz Mabel, LOZANO-GUTIÉRREZ, Jorge Luis, SOLÍS-RECÉNDEZ, Emma \\ Perla y RODRÍGUEZ-GARCÍA, Francisco Javier
}

Universidad Autónoma de Zacatecas

ID $1^{\text {er }}$ Autor: Beatriz Mabel, Pacheco-Amigo / ORC ID: 0000-0002-8053-3506, Researcher ID Thomson: P-7758-2016, CVU CONACYT ID: 514725

ID $1^{\text {er }}$ Coautor: Jorge Luis, Lozano-Gutiérrez / ORC ID: 0000-0001-7294-2572, Researcher ID Thomson: P-8291-2016, CVU CONACYT ID: 947027

ID $2^{\text {do }}$ Coautor: Emma Perla, Solís-Recéndez / ORC ID: 0000-0002-1074-9811, Researcher ID Thomson: S-6705- 2018

ID $3^{\text {er }}$ Coautor: Francisco Javier, Rodríguez-García / ORC ID: 0000-0002-8286-7694, Researcher ID Thomson: S-66832018

DOI: $10.35429 /$ JCP.2019.8.3.1.5

Recibido 13 de Marzo, 2019; Aceptado 30 de Junio, 2019

Resumen

El objetivo de la presente investigación es evaluar aspectos cognitivos, motores y emocionales de menores de preescolar con la finalidad de identificar factores de riesgo dentro del desarrollo en la segunda infancia. La problemática de diversos factores influyen de manera directa la cultura escolar, de esta forma, el estudio se basó en evaluaciones mencionadas, con alumnos que presentaban problemas conductuales, con la finalidad de encontrar indicadores que expliquen el tipo de conducta realizada por el menor que ocasiona perturbación en el contexto escolar en el proceso de enseñanza. Para ello, la metodología utilizada fue de corte no experimental de tipo cualitativo/transversal, mediante un muestreo no probabilístico de sujeto tipo. La contribución obtenida dentro de la investigación se observa con datos cuantificables de frecuencia lineal $(92 \%)$, la influencia directa de la conducta inapropiada de los menores relacionados a la violencia doméstica, recurriendo al contexto escolar como medio de protección afectivo y efectivo, no obteniendo datos significativos en áreas cognitivas.

Psicopedagogía, Factor de protección escuela, Violencia familiar

\begin{abstract}
The objective of the present investigation is to evaluate cognitive, motor and emotional aspects of preschool children in order to identify risk factors within the development in the second childhood. The problem of various factors directly influences the school culture, in this way, the study was based on mentioned evaluations, with students who presented behavioral problems, in order to find indicators that explain the type of behavior performed by the minor that causes disturbance in the school context in the teaching process. For this, the methodology used was of a nonexperimental qualitative / transversal type, through a non-probabilistic sampling of the type subject. The contribution obtained within the investigation is observed with quantifiable linear frequency data $(92 \%)$, the direct influence of the inappropriate behavior of minors related to domestic violence, using the school context as a means of affective and effective protection, not obtaining significant data in cognitive areas.
\end{abstract}

Psychopedagogy, School protection factor, Family violence

Citación: PACHECO-AMIGO, Beatriz Mabel, LOZANO-GUTIÉRREZ, Jorge Luis, SOLÍS-RECÉNDEZ, Emma Perla y RODRÍGUEZ-GARCÍA, Francisco Javier. Elementos psicopedagógicos en niños de preescolar y factores de protección. Revista de Pedagogía Crítica. 2019, 3-8: 1-5

\footnotetext{
* Correspondencia al Autor (Correo electrónico: bpachecoamigo@yahoo.com.mx)

$\dagger$ Investigador contribuyendo como primer autor.
} 


\section{Introducción}

La evaluación psicopedagógica, es considerada como el proceso que se sitúa y se analiza dificultades del pupilo dentro de la escuela como también en el interior del aula. Cuya finalidad es proporcionar herramientas pedagógicas para realizar diversas adaptaciones curriculares o en su caso, sugerencias para adaptarse a un mejor desarrollo motor del menor. Como también, puede ser definida como una evaluación permanente y un constante proceso de investigación, intervención que se relaciona con el aprendizaje escolar, de esta misma forma, el diagnóstico psicopedagógico se considera el análisis de las dificultades del alumno dentro del contexto escolar, donde intervendrán mínimo dos profesionales como ejes del análisis del alumno que serán el psicólogo como especialista y el docente que es quien conoce la situación cotidiana del alumno dentro del contexto del aprendizaje.

Bajo la perspectiva psicopedagógica, el diagnóstico se dirige a un análisis de diferencias individuales e inicialmente era utilizado para la clasificación de los alumnos tomando en consideración las capacidades, aptitudes e intereses. De esta forma, con el surgimiento de diversas teorías, una de ellas los trabajos realizados por Alfred Binet, quien determinó el primer test de cociente intelectual, permitiendo realizar interpretaciones rígidas obviando las capacidades y la contextualización integral del sujeto y, por otro lado se impone el término de cociente intelectual por Lewis Termina (Legaspi, 1997); pensando que los test de inteligencia podía mediar el aprovechamiento escolar y a la vez ser un buen predictor de conducta cognitiva.

Las evaluaciones de las estrategias educativas implementadas en niveles de preescolar, (Garardobil y Maganto 2013) son diseñadas en base al uso de técnicas específicas de lectura dialogada y demandas de distanciamientos que consiste en:

A. Forma de evaluar el impacto de estrategias educativas en el desarrollo de habilidades lingüísticas y simbólicas del infante mediante la observación y pruebas de diagnosis y seguimiento.

B. Estimación de la práctica de la vida cotidiana por parte de los padres de familia.

C. Identificación de líneas de acción localizadas a las prácticas educativas.

ECORFAN ${ }^{\circledR}$ Todos los derechos reservados
Las áreas de evaluación dentro del diagnóstico psicopedagógico, según Bassedas et col (1989), se organizan en diversas unidades de análisis para poder realizar la evaluación y la intervención adecuada, asimismo, sugiere que el diagnóstico pudiese integrarse a la vez como fase preventiva y remediativa para posibles alteraciones a futuro del menor. Por ello, lo centra en diversos enfoques, uno de ellos es el psicotécnico, donde se integra un enfoque potencial de aprendizaje que son la aplicación de tests estandarizados como base de experimento científico, la inteligencia, las capacidades humanas como CI y niveles de desarrollo, evaluación cuantitativa, la conducta como manifestación indirecta de variables complementarias, análisis de unidades morales y una normatividad de la evaluación integrada como base de referencia, todo ello valorado desde una perspectiva de disminución de factores de riego (Pacheco, Cabral y Lozano, 2017).

Para ello, es importante, dentro del diagnóstico psicopedagógico abarcar el contexto familiar del menor, ya que según Covandonga (1999), hace referencia que el rol de la familia dentro de la educación, como primera parte de la institucionalidad educativa, es la cooperación hacia los padres de familia, como formación educadora complementaria de las conductas ejercidas por los padres del menor, por lo que se hace necesario la integralidad del niño, en un ambiente sano, que pueda propiciar el desarrollo de habilidades individuales dentro de su contexto afectivo familiar; por ello, la estructura y el vínculo familiar, como factor social, ejercerá un modelo de aprendizaje aún mayor que los propios de la institucionalización educativa.

La estructura familiar, como principal fuente de socialización aprendizaje de conducta, interviene en la articulación de elementos de desarrollo del infante, mismos, que ayudan a ser precursores de funcionalidad del menor, ..."La familia tiene que relacionarse con un sistema nuevo, bien organizado y de gran importancia. Toda la familia debe elaborar nuevas pautas: cómo ayudar en las tareas escolares; determinar quién debe hacerlo; las reglas que se establecerán sobre la hora de acostarse; el tiempo para el estudio y el esparcimiento, y las actitudes frente a las calificaciones del escolar" (Minuchin, 1980, p.39). 
Mismas relaciones que ayudan a mantener un efecto regulador de la conducta del niño preescolar, manteniendo límites claros y mensajes que ayuden adecuar pensamiento constructivo ante la educación y el contexto social inmediato.

\section{Metodología}

La presente investigación fue elaborada de forma sistemática con la finalidad de integrar de manera apropiada un diagnóstico psicopedagógico en infantes de pre escolar de manera estructurada mediante técnicas de recopilación de datos. Por consiguiente, la misma se establece bajo una perspectiva de carácter socio educativo examinada por medio de una postura positivista, estableciendo lineamientos cuantitativos de comportamiento y desde la fenomenología se especificaron las cualidades de la realidad estudiada que brindó los elementos de objetividad del estudio.

Con lo descrito se establece el tipo de estudio de corte transversal con enfoque de corte mixto que permitió recolectar datos cualitativos mediante la evaluación de escalas estimativas, en las que se integraron patrones previamente evaluado por pruebas psicométricas estandarizadas a la población mexicana. Asimismo, el enfoque cualitativo permitió valorar categorías conductuales del menor.

El muestreo se llevó a cabo mediante una selección muestral de tipo no probabilística de tipo intencionada (sujeto/tipo), por lo que se realizó un método de tipo no aleatorio y la representatividad de la investigación fue en zona institucional educativa con un total de 202 menores. Se desarrollaron parámetros de inclusión derivadas de las mismas instituciones que fueron: alumnos regulares, sexo indistinto, alumnos de preescolar, programa académico escolarizado y canalización de menores por parte del área psicopedagógica.

Para alcanzar los objetivos trazados de la investigación se llevó a cabo la integración de un diagnóstico psicopedagógico en un promedio de 7 meses, donde se establecieron tres unidades de análisis que fueron los alumnos, padres de familia y docentes.
Los instrumentos que se utilizaron en la investigación fueron: test Viso-Motor de Laureta Bender, Cuestionario de Evaluación a Víctima de violencia Intrafamiliar,Test de Matrices Progresivas versión color, test de la Familia, Test de entrevista estructurada y semi estructurada a padres de familia a docentes y técnicas de observación (Herrero, 1996).

Con los resultados arrojados se integraron categorías específicas que fueron:

Categoría 1: Ámbito socioafectivo: Problemas conductuales (Agresividad, Retraimiento, Hiperactividad).

Categoría 2: Ámbito Cognoscitivo: Cociente intelectual.

Categoría 3: Ámbito psicomotor ; Maduración visomotora.

\section{Resultados}

Integrando las categorías mencionadas con anterioridad se presenta la gráfica con una $n=$ 202 menores, arrojando los siguientes datos:

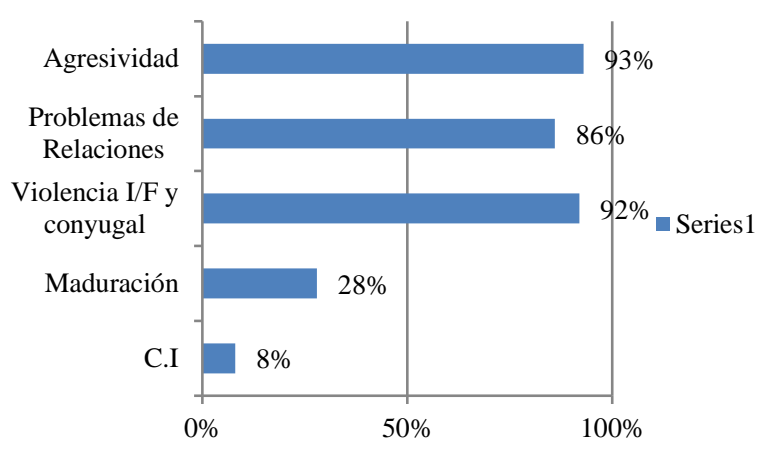

Gráfica 1 Índicadores

Nota: Se muestran los porcentajes de frecuencia lineal de los indicadores sobresalientes de las tres categorías que alcanzaron el $93 \%$ de conductas agresivas, $92 \%$ de violencia intra familiar y conyugales, $86 \%$ problemas de relaciones sociales, $28 \%$ de maduración y $8 \%$ de cociente intelectual.

\section{Conclusiones}

Al integrar las diferentes evaluaciones de la muestra establecida se concluye que: 
1. Existe una alta incidencia relacionada con los problemas académicos de los menores en conjunto con los problemas conyugales de los padres, identificándose como uno de los factores predisponentes , exteriorizando conductas dentro del contexto educativo mismos que son desfavorables para el aprendizaje, refiriendo conductas de agresividad, retraimiento e hiperatividad.

2. Se pudo detectar que sólo el $8 \%$ de la muestra estudiada tiene un cociente intelectual por debajo del término medio y a su vez alcanza un estándar de maduración solamente el $28 \%$ de los menores estudiados, por ello, la importancia del medio contextual ambiental inmediato es el regulador de conductas inmediata, pero en cuanto al C.I y maduración no exigen una significancia estricta a nivel porcentual.

3. El $92 \%$ de la muestra estudiada refiere ambientes familiares inadecuados para el desarrollo del infante, mismo porcentaje que hace alusión a violencia intrafamiliar y conyugal dentro del contexto familiar.

4. El 86\% de los niños presentan problemas para relacionarse con otras personas y a su vez, presentan dificultad de expresar sentimientos, dificultad que se aborda de manera directa a los docentes de pre escolar quienes deben brindar un mayor apoyo a los menores con estas características.

Por tanto, con los elementos expuestos con anterior, se concreta la investigación a nivel general que el $93 \%$ de la muestra manifiesta agresividad por medio de los problemas conyugales observados dentro del hogar, mismas características que hacen vulnerables a los infantes por la etapa en la que se encuentran en el desarrollo. Por ello, la importancia de realizar investigaciones de corte pre escolar relacionadas al diagnóstico psicopedagógico para poder establecer estrategias de tipo preventivas y remediativas en problemas de contextos educativos.
De esta manera se confirma que el factor educativo, es un elemento de protección afectivo -vincular del alumno, disminuyendo factores de ansiedad de los menores al brindar elementos socioemocionales bajo una perspectiva de integralidad basándose, entre otros, en la autonomía y autocontrol.

\section{Sugerencias y limitaciones}

Una de las limitaciones que tuvo la investigación fue el muestreo de tipo no probabilístico de sujeto tipo, que tenían problemas escolares dentro del aula, por lo que, es pertinente precisar que sería factible que en otras investigaciones se pudiera efectuar una descripción y comparación de familias y organización académica de los menores de preescolar bajo una perspectiva de corte longitudinal.

Establecer factores de promoción que ayuden a proteger y generar diversos programas preventivos, dirigido a la convivencia escolar específicas de áreas de fundamentación académica preescolar. Con la finalidad de generar condiciones académicas institucionalizadas, que disminuyan vulnerabilidades familiares que predisponen al menor a desarrollar factores de riesgo $\mathrm{y}$, por consiguiente, el organizar ambientes protectores que eviten comportamientos inadecuados dentro y fuera del hogar, favoreciendo así instancias académicas impactando en la formación integral del alumno.

La identificación temprana de conductas inapropiadas en los preescolares e identificar escenarios de riesgo; suman alternativas de atención primaria de salud mental y contextos escolares abordando áreas de docencia y padres de familia, que auxilian el programa de convivencia escolar desarrollada por la Secretaría de Educación Pública.

\section{Referencias}

Bassedas, E., Huguet, T., Marrodan, M., Oliván, M., Planas, M., Rossell, M., Seguir, M y Vilella, M. (1989) Intervención Educativa y diagnóstico psicopedagógico. Paidós: España.

Herrero, L. (1996). La importancia de la observación en el proceso educativo. Paidos:México. 
Legaspi, A. (1997). Pedagogía preescolar. Pueblo y Educación. Cuba

Garardobil, M y Magnate, C. (2013). Padres y maestros: Problemas emocionales y de la Conducta. México.

Minuchin, S. (1980). Familias y Terapia familiar. Gedisa: España.

Pacheco, B., Cabral, Ma. \& Lozano, J. (2017) Childhood obesity and quality of live: psychosocial risks in children aged 6 to 12 years.Internacional Journal for Research in Social Sciencie And Humanities. Volume 3 Issue 12. USA 